\title{
El emprendimiento dentro de las mallas curriculares en las facultades de administración de la ciudad de Quito. Un estudio exploratorio de la realidad académica actual
}

\section{Entrepreneurship as part of the curricula meshes in the business faculties in Quito. An exploratory study of the current academic reality}

Hallo Alvear, Fernando

Salvador Tamayo, Estefanía

Universidad Internacional del Ecuador, Ecuador

Autor por correspondencia: rohalloal@uide.edu.ec, essalvadorta@uide.edu.ec

Fecha de recepción: 27 de febrero de 2018 - Fecha de aceptación: 15 de agosto de 2018

Resumen: Las instituciones de educación superior IES están regidas por leyes y normativas promulgadas para garantizar una formación profesional de calidad con pertinencia y articuladas a las necesidades de transformación y participación social; dicha formación busca cumplir con un perfil de egreso específico de acuerdo a cada área del conocimiento, en la administración constituye el usar los elementos administrativos para diseñar, modelar y generar procesos en las organizaciones dentro de un mercado cambiante que satisfagan las necesidades de los consumidores generando utilidad a los accionistas y/o a la sociedad. Sin embargo, los profesionales que egresan de las instituciones de educación superior pasan a formar parte de mundo laboral en relación de dependencia o de manera independiente, siendo éstos últimos los que generan emprendimientos, muchos de ellos considerados "golondrinas" por su corto tiempo de permanencia en el mercado. La presente investigación busca llegar a conclusiones diagnósticas de manera cuantitativa acerca de la cantidad de asignaturas en las mallas curriculares de las facultades de administración de universidades, tanto públicas como privadas en la ciudad de Quito, que desarrollan habilidades para el emprendimiento en los estudiantes. Esta, se vuelve la primera parte de una investigación que tiene como objetivo final diagnosticar las variables que han llevado a que los emprendimientos continúen en el tiempo, identificando similitudes que puedan servir como base para los nuevos emprendedores.

Palabras Claves: emprendimiento; facultades de administración; instituciones de educación superior; mallas curriculares

Abstract: The institutions of higher education IES are governed by laws and regulations promulgated to guarantee quality professional training with relevance and articulated to the needs of transformation and social participation; This training seeks to meet a specific egress profile according to each area of knowledge, in administration is to use the administrative elements to design, model and generate processes in organizations within a changing market that meet the needs of consumers generating Shareholders and / or society. However, professionals who graduate from higher education institutions become part of the labor market in a dependency or independent relationship, the latter of which generate entrepreneurship, many of them considered "swallows" due to their short time of permanence in the market. The present research seeks to reach quantitative conclusions on the number of subjects in the curricular meshes of the public and private university administration faculties in the city of Quito, which develop students' entrepreneurship skills.

Key words: entrepreneurship; management faculties; institutions of higher education; curricula 


\section{Introducción}

El conocimiento y la innovación constituyen instrumentos fundamentales para alcanzar el desarrollo sostenible de las personas y las sociedades, dicho desarrollo debe ser equitativo e inclusivo en una nación. En este sentido, (SENPLADES, 2014) expresa que:

La sociedad de plenas capacidades, emancipación y autonomías, en donde el desarrollo de los ciudadanos se vuelve como una herramienta fundamental para vivir dignamente, expandiendo sus capacidades y creando oportunidades mediante el conocimiento y la innovación, a fin de satisfacer más allá de sus necesidades mínimas.

Es conocido que los emprendimientos incentivan la producción, generan fuentes de empleo e incrementan la circulación del dinero en una nación; De acuerdo a (Botella \& Suárez, 2012), la innovación para el desarrollo en América Latina está por debajo de la media regional, tomando en cuenta a su población, contribución económica y nivel de desarrollo; sin embargo, según el reporte del Global Entrepreneurship Monitor (2015), que es uno de los proyectos de investigación con más renombre a nivel mundial en temas relacionados a emprendimiento y donde participan 62 países con economías en distintas etapas de desarrollo pero que comparten las motivaciones principales para impulsar nuevos negocios, basados en el Índice de Actividad Emprendedora Temprana (TEA) obtuvo que 1 de cada 3 personas adultas habían realizado actividades en relación a la creación de nuevos negocios, y que el 33,6\% ya tenían un negocio con una antigüedad de hasta 42 meses.

Por otro lado, el mismo informe señala que aquellos negocios que han continuado operando por encima del tiempo establecido (42 meses), han sido únicamente el 17,4\%. Con respecto al enfoque de los negocios, los emprendimientos nuevos alcanzan un $76 \%$ y los negocios establecidos en el tiempo, llegan al 51\%. Dichos negocios forman parte del mercado del comercio, especialmente el doméstico.

Es importante recalcar que este mismo reporte establece una caracterización de los emprendimientos o negocios de la siguiente manera:

- El 25,9\% de negocios son nacientes

- El 9,8\% de negocios son nuevos

- El 10,3\% son personas que crearon su negocio por necesidad

- El 23,1\% de los negocios surgieron por aprovechamiento de oportunidades

Pero la temporalidad de estos negocios se convierte en un problema por resolver, puesto que esto provoca una desaceleración en la dinamización económica, muchas de las veces la inversión no es recuperada, se desincentiva futuros emprendimientos con impacto social y económico, entre otras consecuencias.

Analizando la realidad nacional, Ecuador forma parte de los 15 países con mayor probabilidad de iniciar emprendimientos (GEM, 2014); sin embargo, a pesar de las fortalezas encontradas, como por ejemplo la capacidad de detectar y aprovechar las oportunidades en el mercado, se encuentran también grandes debilidades al momento de comenzar nuevos negocios, 
entre las cuales están el insuficiente capital para la inversión inicial y la falta de conocimiento debido a la escasa capacitación formal al que acceden los emprendedores.

Cabe destacar que Ecuador despunta junto a Zambia y Nigeria como los países con más emprendimientos, entre una muestra de 69 países. En contraste, los países menos emprendedores que son Japón, Francia e Italia. (GEM; 2013)

Por otro lado, es necesario señalar que en el Ecuador en los últimos años los emprendimientos han sido considerados como la solución a los problemas derivados de los altos niveles de desempleo en el país. Según datos publicados por el INEC, en el 2015, el desempleo en la ciudad de Quito bordeaba el 4,9\% de la Población Económicamente Activa (PEA); para el año 2016, el porcentaje se duplicó, con un incremento del 9,1\%. Adicionalmente, en el caso del subempleo, la situación fue más fuerte, con un aumento del 5,5\% en el año 2015 al $13 \%$ en el 2016.

Como resultado de la situación actual en el tema de desempleo que atraviesa el país, el emprendimiento surge como una motivación para el desarrollo profesional de los ciudadanos. La Agencia de Promoción Económica CONQUITO, señala que durante el segundo semestre del año pasado, se incrementó un 35\% las solicitudes de personas en busca de capacitaciones para emprender sus propios negocios. Este porcentaje se ha incrementado en el 2017, en lo que va del año se puede evidenciar un $15 \%$ adicional. Se destaca la importante participación de esta Agencia para el desarrollo económico de Quito, puesto que impulsan 7 diferentes áreas de soporte al emprendimiento. De acuerdo a datos publicados por la misma Agencia CONQUITO (2017), durante el año pasado capacitó a más de 15.000 personas, dio asistencia técnica a 10.000 personas aproximadamente, e impulsó a 1.300 nuevos negocios de emprendedores. El dato más sorprendente refleja la ayuda a más de 20.000 emprendedores durante el 2016.

De acuerdo a (World Bank, 2016), en su reporte anual Doing Business, el ciclo de vida de un negocio debe pasar por algunas etapas; en este sentido, Ecuador ha facilitado el proceso para crear un negocio con varias herramientas para el impulso de los emprendimientos. Además, obtuvo el rendimiento regional más bajo en relación al tiempo que se demora un negocio para resolver problemas de insolvencia.

Es importante señalar que, en 2015, el índice en relación a la formación académica de los negociantes fue la siguiente:

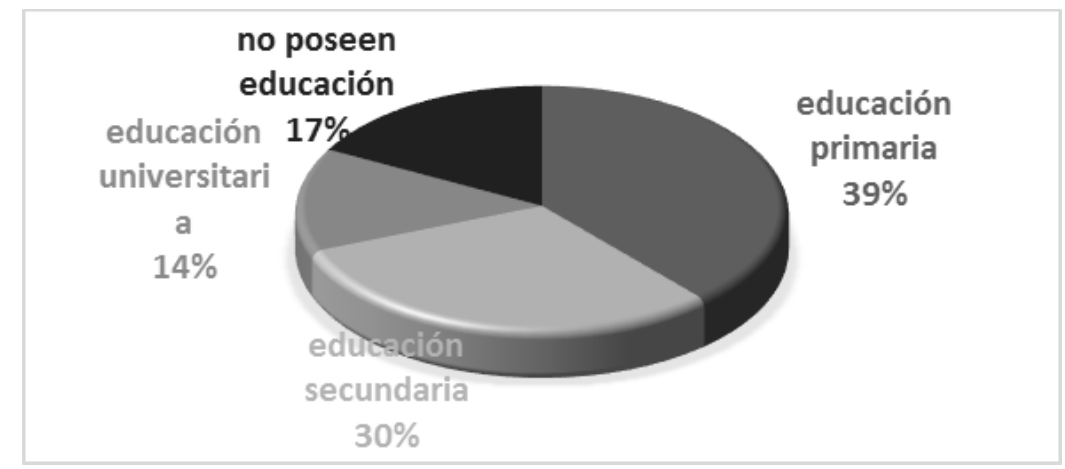

Figura 1. Formación académica de los negociantes - emprendedores en Ecuador 
Fuente: World Bank, 2016

Son cifras que merecen un análisis profundo, debido a que el bajo porcentaje de formación académica - sobre todo universitaria - puede estar atado al tema de la corta duración de vida de los negocios.

De todas maneras, la tasa de emprendimientos por necesidad en el país se encuentra ubicada por encima de la media del Continente Americano; dentro de los negocios por oportunidad, se encuentran los porcentajes presentados el Figura

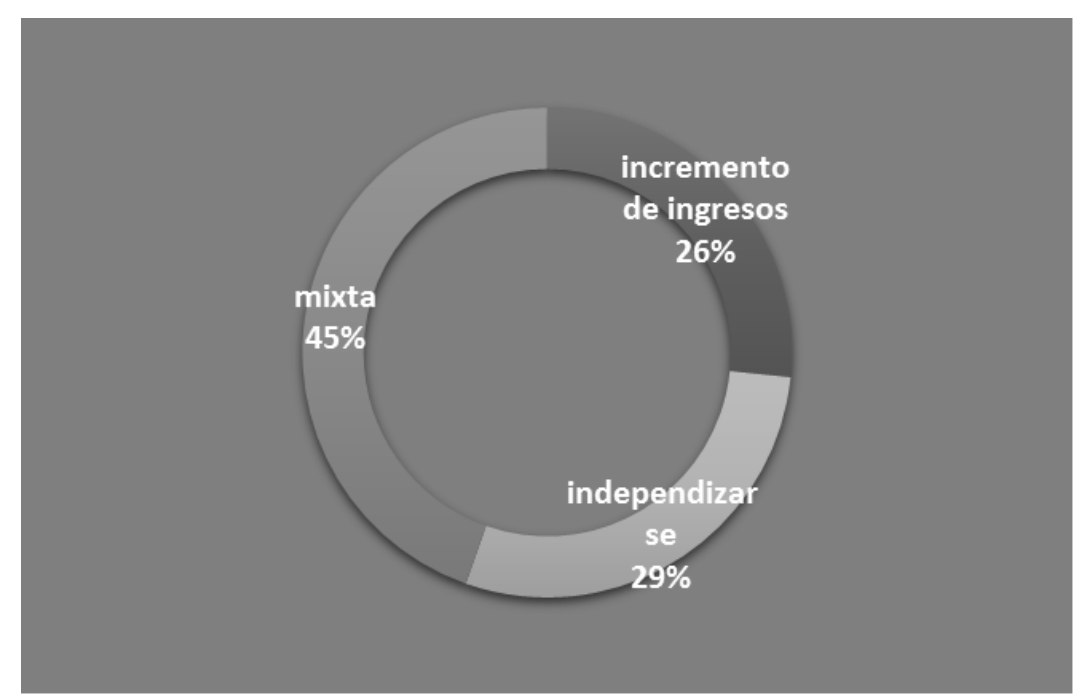

Figura 2. Motivación de los emprendedores en los negocios por oportunidad Fuente: World Bank, 2016

En relación a los elementos que promueven los emprendimientos en el Ecuador, se pueden mencionar siete factores principales:

1. Apoyo financiero

2. Capacidad para emprender

3. Programas de gobierno

4. Políticas gubernamentales

5. Educación y entrenamiento

6. Apertura del mercado

7. Normas sociales y culturales

Dentro de la literatura, se encuentran varios autores que detallan los conductores en temas de emprendimiento, (Shane, Locke, \& Collins, 2003), mencionó que si los factores del medio ambiente permanecen constantes, la motivación personal desarrolla un rol fundamental para el inicio del proceso emprendedor. Por otro lado (Veciana,2005) señaló la importancia de 5 elementos, los cuales incluyen la identificación de la oportunidad a desarrollar, la adquisición de los factores de producción como por ejemplo recursos humanos y materiales; la elección del mercado en el que se desenvolverá el emprendimiento, creación e implementación de estrategias y por último la motivación del negociante. 
Es así que se puede asegurar que la mayoría de autores coinciden en la importancia de la motivación para emprender, adquisición de los recursos necesarios junto con la adaptación de un excelente ambiente de negocios.

Una de las fuentes de financiamiento más utilizadas en el territorio ecuatoriano es el denominado capital de riesgo, el cual es promovido también por el Banco de Ideas. ${ }^{1}$ Además, están los microcréditos impulsados por varios bancos e instituciones del sector popular y solidario como las cooperativas a nivel nacional, sin restar importancia aquellos préstamos informales por parte de familiares o amigos que pueden llegar a un número importante dentro de la proporción del financiamiento.

Posteriormente, al comparar los emprendimientos en el Ecuador con el resto de economías analizadas en el informe del Global Entrepreneurship Monitor, se puede afirmar que la TEA para nuestro país es 2,2 veces mayor que el promedio de las economías de eficiencia. Pero, es interesante tener en cuenta que la motivación principal de los emprendimientos en el Ecuador es mayor en relación a la necesidad, mientras que la motivación de las economías de eficiencia se relaciona con el aprovechamientos de nuevas oportunidades, deseo de incremento de ganancias y altas expectativas de crecimiento laborales.

(López, Santos, \& Bueno, 2004) caracterizan al emprendedor exitoso como una persona altamente motivada por ciertos factores en los que incluye la necesidad, y la percepción del beneficio económico del negociante. Además de la iniciativa y la energía combinada con trabajo, compromiso, autocontrol y las ganas de alcanzar nuevos retos.

En este sentido, se puede entender que la innovación es un proceso dinámico que depende de varios factores, entre los cuales se encuentran las instituciones, el capital humano y las estructuras productivas generadas a través de los mismos. Este tipo acciones determinan las actividades competitivas de las economías industriales avanzadas, y por ende también generan oportunidades en las economías emergentes (Robayo, 2016). Además, deber contener una filosofía en la que se resalte un marco conceptual para la acción en la que el término emprendimiento es dominante como fortaleza a desarrollar en la formación del estudiante y futuro egresado (Muñoz \& Martínez, 2011).

Algunas de las instituciones que deben apoyar al emprendimiento de la población, entre otras, son las universidades, puesto que se convierten en las generadoras de conocimiento. En Ecuador existen universidades públicas y privadas que están reguladas por la Ley Orgánica de Educación Superior (LOES), algunas de ellas cuentan con el apoyo gubernamental desde el punto de vista del presupuesto anual designado a la educación superior en el sector público, en contraste que las universidades particulares designan parte de su presupuesto autofinanciado a este rubro.

\footnotetext{
${ }^{1}$ La Secretaría de Educación Superior, Ciencia, Tecnología e Innovación por medio de la Subsecretaría de Innovación Tecnológica, ha desarrollado el primer "Banco de Ideas" en el Ecuador que promueve el primer portal interactivo del país en el que cualquier persona natural puede proponer proyectos, investigaciones y prototipos con el objetivo de recibir apoyo y servicios enfocados en su idea innovadora. El valor agregado de este portal se vincula al desarrollo de los componentes creativos, tecnológicos e innovadores de las ideas que sean seleccionadas como ganadoras. (SENESCYT, 2017).
} 
El Consejo Ecuatoriano de Acreditación y Aseguramiento de la Calidad de la Educación Superior (CEAACES) es el organismo público que tiene como misión la rectoría de la política pública en función del aseguramiento de la calidad y excelencia en la educación superior por medio de procesos de evaluación, acreditación, y categorización de las Instituciones de Educación Superior (IES).

De acuerdo al Consejo de Evaluación, Acreditación y Aseguramiento de la Calidad de la Educación Superior (CEAACES, 2017):

En septiembre de 2015, el Consejo de Evaluación, Acreditación y Aseguramiento de la Calidad de la Educación Superior-CEAACES- inició en un proceso de Evaluación acreditación y recategorización institucional en el que participación 13 Instituciones de Educación Superior -IES-.

Del total de universidades evaluadas, 12 participaron en la recategorización de forma voluntaria y solo para la Universidad de las Fuerzas Armadas (ESPE) el proceso fue obligatorio porque no fue incluida en la valoración del 2013.

El resultado de lo mencionado fue el categorizar a las instituciones de educación superior con una escala entre la categoría A y la D. el listado se lo encuentra en la.

Tabla 1. Listado de instituciones acreditadas - CEAACES 2016

\begin{tabular}{|c|c|c|}
\hline Categoría & Institución de Educación Superior & \\
\hline $\mathrm{A}$ & $\begin{array}{ll}\text { - } & \text { Escuela Politécnica Nacional } \\
\text { - } & \text { Escuela Superior Politécnica del Litoral } \\
\text { - } & \text { Universidad San Francisco de Quito } \\
\text { - } & \text { Universidad de Cuenca } \\
\text { - } & \text { Universidad de las Fuerzas Armadas (ESPE) } \\
\text { - } & \text { Universidad de Especialidades Espíritu Santo } \\
\text { - } & \text { Facultad Latinoamericana de Ciencias Sociales } \\
\text { - } & \text { Universidad Andina Simón Bolívar }\end{array}$ & \\
\hline B & $\begin{array}{l}\text { Tabla } 1 \text { de Instituciones Acreditadas - CEAACES } 2016 \text { (continuación) }\end{array}$ & Listado \\
\hline & $\begin{array}{ll}\text { - } & \text { Escuela Superior Politécnica de Chimborazo } \\
\text { - } & \text { Pontificia Universidad Católica del Ecuador } \\
\text { - } & \text { Universidad Casa Grande } \\
\text { - } & \text { Universidad Católica de Santiago de Guayaquil } \\
\text { - } & \text { Universidad Central del Ecuador } \\
\text { - } & \text { Universidad del Azuay } \\
\text { - } & \text { Universidad Estatal de Milagro } \\
\text { - } & \text { Universidad Nacional de Loja } \\
\text { - } & \text { Universidad Particular Internacional SEK } \\
\text { - } & \text { Universidad Politécnica Salesiana } \\
\text { - } & \text { Universidad Técnica de Ambato } \\
\text { - } & \text { Universidad Técnica del Norte } \\
\text { - } & \text { Universidad Técnica Estatal de Quevedo } \\
\text { - } & \text { Universidad Técnica Particular de Loja } \\
\text { - } & \text { Universidad Tecnológica Empresarial de Guayaquil }\end{array}$ & \\
\hline
\end{tabular}


- Universidad Tecnológica Equinoccial

- Universidad Tecnológica Indoamérica

- Universidad de los Hemisferios

- Universidad Estatal Amazónica

- Universidad Politécnica del Carchi

- Universidad Iberoamericana

- Universidad Técnica de Manabí

- Universidad de las Américas

- Universidad Internacional del Ecuador

- Universidad de Guayaquil

- Universidad Técnica de Machala

- Instituto de Altos Estudios Nacionales

Tabla 1

de Instituciones Acreditadas - CEAACES 2016 (continuación)

Listado

- Escuela Superior Politécnica Agropecuaria de Manabí

- Universidad de Especialidades Turísticas

- Universidad del Pacífico Escuela de Negocios

- Universidad Estatal de Bolívar

- Universidad Laica Vicente Rocafuerte de Guayaquil

C

- Universidad Metropolitana

- Universidad Nacional del Chimborazo

- Universidad Regional Autónoma de los Andes

- Universidad Técnica de Babahoyo

- Universidad Tecnológica Israel

- Universidad Estatal Península de Santa Elena

- Universidad Particular San Gregorio de Portoviejo

- Universidad Tecnológica ECOTEC

- Universidad Técnica de Cotopaxi (Institución en situación de irregularidad académica)

- Universidad Estatal del Sur de Manabí

- Universidad de Otavalo

D $\quad$ Universidad Agraria del Ecuador

- Universidad Laica Eloy Alfaro de Manabí

- Universidad Técnica Luis Vargas Torres de Esmeraldas

- Universidad Católica de Cuenca

Nota: Recuperado de http://www.ceaaces.gob.ec/sitio/acreditacion-y-categorizacion/ mayo de 2017.

Los criterios que fueron tomados en cuenta son: academia, ambiente estudiantil, organización, recursos e infraestructura y vinculación con la sociedad. El proceso del año 2015, contó con la participación de 7 comités de evaluación y 28 evaluadores nacionales e internacionales, los cuales revisaron la información y constataron los avances en cada una de las instalaciones. Esta revisión se fundamentó en 44 indicadores, 25 cuantitativos y 19 cualitativos.

Con lo mencionado anteriormente, los emprendimientos que nacieron en las universidades dinamizan la economía al momento de incentivar la producción y generar fuentes de empleo que, a su vez, incrementan la circulación del dinero en un país; por tal motivo, es imprescindible analizarlos para conocer los factores internos y externos que han permitido su permanencia en el mercado y sacar de esta manera conclusiones que permitan emularlos. 
En cuanto a los niveles de formación de Educación Superior, el presente estudio se basa en identificar las asignaturas de las mallas curriculares relacionadas al emprendimiento dentro de los estudios de tercer nivel. Dentro del Anexo 1 de la LOES, se presenta la codificación de los campos de conocimiento; Administración se encuentra en el 4to campo amplio, posteriormente el 1er campo específico muestra la categoría de Educación Comercial y Administración, y el 3er campo detallado Administración, categoría B Administración de Empresas en carreras de grado con el título de Licenciado en Administración de Empresas.

\section{Metodología}

Para la elaboración del presente trabajo se tomó como base la información presentada en la investigación expuesta en el Congreso Internacional de Investigación en Ciencias Administrativas y Económicas CIICAE-UIDE 2017, realizado en Loja-Ecuador, durante los días 17 al 19 de mayo; la actualización de la misma está plasmada en este documento.

Una de las metodologías utilizadas en la presente investigación es el método inductivo; mismo que permite llegar a conclusiones generales desde enunciados observacionales particulares investigados, para este trabajo, en función del requerimiento en fuentes secundarias; se comenzó con la observación del hecho tratado, analizándolo en su contexto y concluyendo en una definición concreta que es la presentada en el actual trabajo.

El enfoque está en analizar las mallas curriculares de las universidades en la ciudad de Quito que oferten la carrera de Administración de Empresas con el fin de determinar de manera cuantitativa las asignaturas que guarden relación con el emprendimiento o innovación en función del total de materias dictadas en la carrera. No se toma en cuenta la diferenciación de algunas IES sobre créditos y horas académicas ni el perfil de egreso, simplemente se basa en el criterio no técnico del nombre de la materia que debe guardar relación al emprendimiento o a la innovación.

Además, permitió presentar hechos relevantes comprendiendo el amplio espectro aplicativo del tema planteado. Las fuentes secundarias investigadas fueron las páginas de los organismos de control gubernamental de la Educación Superior, las mallas académicas de las universidades que forman parte del segmento investigado, autores que hablan sobre el tema de emprendimiento y educación superior con el fin de apalancarse de teoría adecuada.

\section{Resultados}

Se pudo determinar que las universidades de la ciudad de Quito que ofertan la carrera de Administración de Empresas, sin excluir por su modalidad de estudios, son las que se muestran en la Tabla 2.

Tabla 2. Universidades de la ciudad de Quito con oferta de la carrera de Administración de Empresas

\begin{tabular}{ll}
\hline Universidad & Categoría CEAACES \\
\hline Escuela Politécnica Nacional EPN & A \\
Universidad San Francisco de Quito USFQ & A \\
\hline
\end{tabular}




\begin{tabular}{ll}
\hline Universidad de las Fuerzas Armadas ESPE & A \\
Universidad Tecnológica Indoamérica UTI & B \\
Universidad Internacional del Ecuador UIDE & B \\
Universidad Tecnológica Equinoccial UTE & B \\
Pontificia Universidad Católica del Ecuador PUCE & B \\
Universidad Técnica Particular de Loja UTPL & B \\
Universidad Politécnica Salesiana UPS & B \\
Universidad Central del Ecuador UCE & B \\
Universidad de las Américas UDLA & B \\
Universidad de los Hemisferios UHemisferios & B \\
Universidad Metropolitana UMET & C \\
Universidad Tecnológica Israel UIsrael & C \\
\hline
\end{tabular}

Nota: Fuente Investigación propia.

Es decir, tres universidades de categoría A, nueve de categoría B y dos de la C ofertan la carrera de Administración de Empresas en Quito. Algunas de ellas en distintas modalidades, presencial, semipresencial y a distancia o virtual.

Analizando las mallas curriculares de las universidades antes mencionadas y teniendo en cuenta únicamente las materias relacionadas al emprendimiento e innovación como tal, se obtuvo lo siguiente por universidad y categorías.

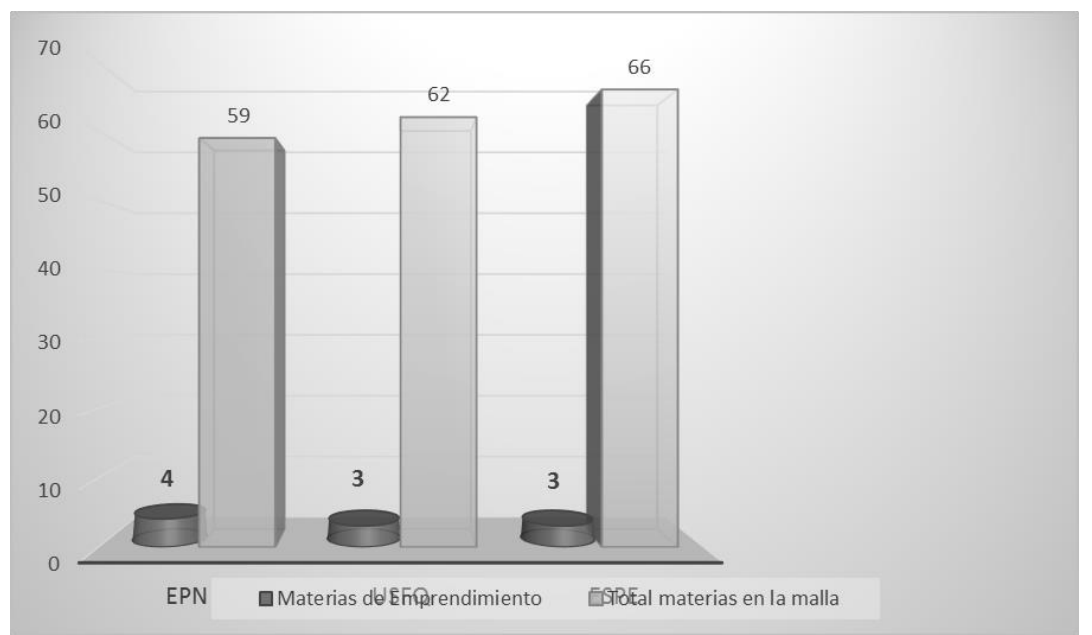

Figura 3. Cantidad de materias relacionadas al emprendimiento en universidades de Categoría A Fuente: Investigación propia

En la Escuela Politécnica Nacional 4 de 59 materias (7\%), en la Universidad San Francisco de Quito existen 3 materias de 62 (5\%), y en la Universidad de las Fuerzas Armadas hay 3 de 66 materias dictadas en la malla $(5 \%)$. 


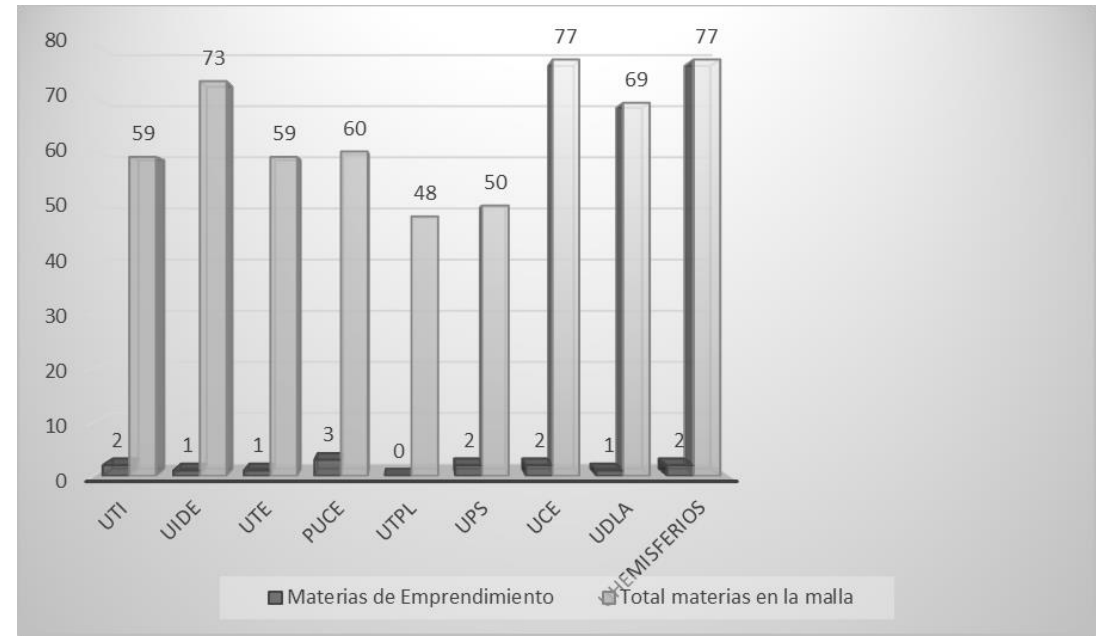

Figura 4. Cantidad de materias relacionadas al emprendimiento en universidades de Categoría B Fuente: Investigación propia

En la Universidad Tecnológica Indoamérica UTI existen 2 de 59 materias (3\%), en la Universidad Internacional del Ecuador existe 1 materia de 73 (1\%), en la Universidad Tecnológica Equinoccial UTE hay 1 de 59 materias dictadas en la malla (2\%), en la Pontificia Universidad Católica del Ecuador PUCE existen 3 materias de 59 (5\%), en la Universidad Técnica Particular de Loja UTPL no hay materias relacionadas de 48 existentes $(0 \%)$, en la Universidad Politécnica Salesiana hay 2 de 50 materias (4\%), en la Universidad Central del Ecuador ofertan 2 de 77 materias (3\%), en la Universidad de las Américas hay 1 de 69 materias (1\%), y por último en la Universidad de los Hemisferios existen 2 materias de 77 en total de la malla $(3 \%)$.

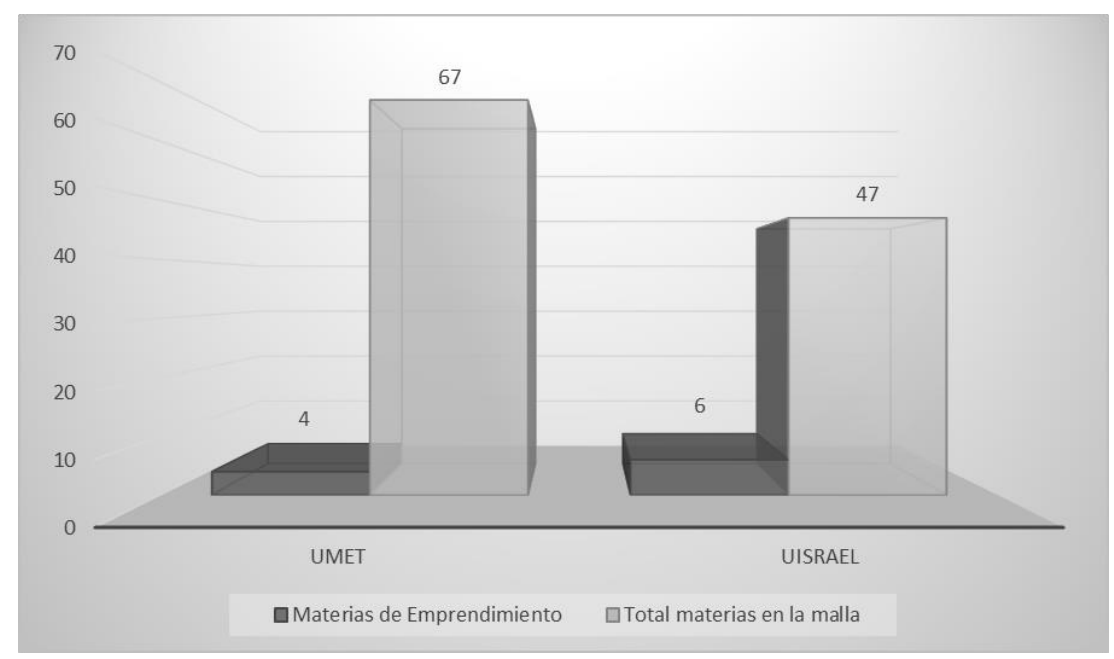

Figura 5. Cantidad de materias relacionadas al emprendimiento en universidades de Categoría $C$ Fuente: Investigación propia

En la Universidad Metropolitana hay 4 de 67 materias (6\%) y en la Universidad Israel existen 6 materias de $47(13 \%)$.

\section{Conclusiones}


Con lo investigado, y teniendo en cuenta que el presente trabajo es parte de un proyecto de investigación que busca analizar los emprendimientos generados por estudiantes de las facultades de administración de las universidades de Quito en el periodo 2006 - 2016 con el fin de determinar un esquema de factores de éxito comunes, se presentan las siguientes conclusiones:

El Consejo Ecuatoriano de Acreditación y Aseguramiento de la Calidad de la Educación Superior CEAACES, categorizó a las instituciones de educación superior en letras desde la A hasta la $\mathrm{C}$, siendo la $\mathrm{A}$ las que cumplieron con todos los parámetros exigidos en la Ley Orgánica de Educación Superior LOES.

En la ciudad de Quito existen catorce universidades que ofertan la carrera de Administración de Empresas con modalidades como presencial, semipresencial y a distancia, las mismas que se encuentran categorizadas en A, B y C, conforme lo expuesto anteriormente. Estas son: Categoría A - tres universidades, Categoría B - nueve universidades, y por último, en la Categoría C están dos.

El estudio presenta los siguientes resultados tomando en cuenta el cien por ciento de las mallas curriculares analizadas en cada universidad y de cada categoría. Los resultados son: Categoría A refleja temas de emprendimiento o innovación en su malla curricular en el seis por ciento de sus asignaturas. Para las de Categoría B, el porcentaje se reduce a un dos por ciento; y las de Categoría $\mathrm{C}$, el porcentaje se incrementa nuevamente al diez por ciento.

Dentro de la Categoría C, se encuentra una universidad cuya malla curricular muestra trece por ciento de asignaturas enfocadas a temas de emprendimiento e innovación. Este dato puede analizarse desde el punto de vista que una universidad puede tener un mayor enfoque a temas de emprendimiento, independientemente de su categorización; es decir, esto será uno de los puntos de partida para las siguientes fases del proyecto de investigación mencionado.

El cien por ciento de las universidades que se encuentran en la Categoría A y C, tienen por lo menos una asignatura enfocada a emprendimiento o innovación, en contraste a lo que se presenta con una universidad de la de categoría B en la que no se encontró ninguna materia relacionada.

Las mallas de las universidades, de acuerdo a su modalidad de estudio, son distintas en su estructura; provocando de cierta forma una complicación en el análisis por institución educativa pero que no limita la exploración de los datos.

El porcentaje promedio de todas las universidades que ofertan la carrera de administración de empresas en la ciudad de Quito, se encuentra en el cuatro por ciento. Sin embargo, mencionar si esta cifra es baja o alta no es adecuado en esta fase del proyecto hasta contrastar con más información que permita llegar a una conclusión.

\section{Logros y proyecciones a futuro}


El presente trabajo forma parte de un proyecto de investigación cuyo objetivo principal es identificar los emprendimientos que han tenido éxito en los últimos años y que han salido de las aulas universitarias.

Los resultados de la presente investigación, dan una pauta necesaria para entender el enfoque que buscan las Instituciones de Educación Superior en la formación de los profesionales en el área administrativa.

\section{Bibliografía}

Consejo de Evaluación, Acreditación y Aseguramiento de la calidad de la Educación Superior. (2017). Categorización de Universidades. Recuperado el 04 de Mayo de 2017, de http://www.ceaaces.gob.ec/sitio/categorizacion-de-universidades/

Conquito (2017). Emprendimiento e innovación. Recuperado el 04 de mayo de 2017, de http://www.conquito.org.ec/emprendimiento-e-innovacion/

ESPOL. (2013). Global Entrepreneurship Monitor: Informe GEM 2012. Recuperado de http://www.espae.espol.edu.ec/images/FTP/ReporteGEM2012.pdf

ESPOL. (2015). Global Entrepreneurship Monitor: Informe GEM 2014. Recuperado de http://www.espae.espol.edu.ec/images/documentos/publicaciones/libros/gemecuador2014 .pdf

ESPOL. (2016). Global Entrepreneurship Monitor: Informe GEM 2015. Recuperado de: http://www.espae.espol.edu.ec/images/documentos/publicaciones/libros/GemEcuador201 5.pdf

González, M. (04 de Mayo de 2017). ¿Cuáles son las nuevas categorías de las universidades ecuatorianas tras la recategorización? Diario El Comercio

López, I., Santos, B., \& Bueno, Y. (2004). Las Dimensiones del Perfil del Emprendedor: Contraste empírico con emprendedores de éxito. El emprendedor innovador y la creación de empresas de $I+D+I$.

Muñoz, O., \& Martínez, W. (2011). Universidad y emprendimiento, aportes para la formación de profesionales emprendedores. Cuadernos de Administración. Universidad del Valle.

Robayo, P. (2016). La innovación como proceso y su gestión en la organización: una aplicación para el sector gráfico colombiano. Suma de Negocios.

Secretaría Nacional de Educación Superior, Ciencia, Tecnología e Innovación. (2 de Mayo de 2017). Banco de Ideas. Innovación Social. Recuperado el 04 de mayo de http://www.bancodeideas.gob.ec/site/index

Secretaría Nacional de Planificación y Desarrollo. (2014). Plan Nacional del Buen Vivir 20132017. Quito: Editorial El Conejo. 
Shane, S., Locke, E., \& Collins, C. (2003). Entrepreneurial Motivation. Human Resource Management Review.

Veciana, J. (2005). La creación de empresas. Un enfoque gerencial. Colección de estudios económicos. Madrid: Editorial La Caixa.

World Bank. (2016). Doing Business Regional Profile 2017 : Latin America and Caribbean. Washington, DC: World Bank. 